\title{
Edge states in coupled periodic dielectric waveguides induced by long-range interaction
}

\author{
R. S. Savelev \\ ITMO University, St. Petersburg 197101, Russia \\ r.savelev@metalab.ifmo.ru
}

PACS 42.79.Gn, 42.82.Et

DOI 10.17586/2220-8054-2018-9-6-716-723

\begin{abstract}
We study the properties of coupled periodic dielectric nanowaveguides and reveal that finite arrays of equally spaced waveguides with certain parameters support edge modes, which originate due to the presence of the long-range interactions between the waveguides. We provide a simple model of the coupled waveguides with next-to-nearest neighbors interaction that captures the main properties of the considered system including the formation of the edge states. The predicted results suggest that the arrays of periodic dielectric nanowaveguides may serve as a fruitful system for studying the discrete coupled systems with long-range interaction and realizing optical metasurfaces with novel functionalities for guiding surface waves.
\end{abstract}

Keywords: periodic waveguides, coupled discrete systems, edge states, long-range interaction.

Received: 19 November 2018

Revised: 6 December 2018

\section{Introduction}

Recently, arrays of coupled dielectric and plasmonic waveguides have been studied in a vast amount of theoretical and experimental works and have been proven to serve as a very convenient platform to study various optical effects such as discrete diffraction [1], observation of topologically protected photonic edge states [2-4] and formation of optical solitons $[5,6]$. The properties of the waveguide arrays usually can be determined very accurately within the tight-binding approximation, which allows one to employ such discrete systems for emulation and direct observation of optical analogues of many effects from solid state physics such as Bloch oscillations [7-9], Zener tunneling [10,11], Anderson localization [12-14] etc [15].

The flexibility of the waveguide arrays in terms of their modulation in transverse or longitudinal direction provides additional versatility to these systems [16]. Such modulation, typically achieved by bending the waveguides or by changing the refractive index in the direction of the waveguide axis changes their propagation constants or interaction constants between the waveguides and leads to such phenomena as dynamic localization and subdiffractive propagation of light [16]. The extreme case of longitudinal refractive index modulation corresponds to the formation of a periodic waveguide that consists of separate parts [17-19]. Such waveguides, composed of individual dielectric nanoparticles with high refractive index, such as silicon, were proposed and theoretically studied few years ago and experimentally realized only recently [20]. The dispersion properties of such nanowaveguides are mostly determined by the optical response of their constituent elements, i.e. dielectric nanoparticles. Extensive studies in this field in the last several years have provided multiple experimental observations of the efficient geometrical tuning of the high-index dielectric nanoparticles [21], which consequently suggests a wide potential in controlling the properties of the nanoparticle waveguides [22].

The presence of the periodicity in a waveguide might bring additional features in the properties of the coupled waveguides system due to several reasons. First, the electromagnetic fields in the nanoparticle waveguides become highly confined, and, therefore, their vectorial nature has to be taken into account. Second, as compared to an array of homogeneous waveguides, where the only parameter is the distance between the waveguides, periodic waveguides can also be shifted relative to each other along their axis. Such a shift results in the formation of a different system with potentially different properties similar to the modulated optical lattices.

In a conventional scenario, the edge modes in coupled discrete systems appear as a result of a perturbation at the boundary of the structure or propagation constant shift in nonlinear regime [23]. However, in systems with complex geometry surface states can exist even without any perturbation. For instance, in an array of curved waveguides, the surface states can appear due to transverse modulation of the waveguides axes, which induces "virtual" perturbation at the boundary of the array [24]. Here, we show that an array of equally spaced alternating nanoparticle waveguides of two types, as schematically shown in Fig. 1, may possess nontrivial eigenmode structure. In particular, we demonstrate that the specially designed array of nanocylinder chains shifted relative 
to each other by half of the period possesses a gap in the spectrum of the eigenstates, which is accompanied by the formation of the edge states in the finite size array, despite the absence of any edge perturbation. We show that the presence of the spectral gap cannot be understood within the conventional tight-binding approximation and requires taking into account at least the next-to-nearest neighbor's interaction.

\section{Polarization properties of individual nanoparticle waveguides}

As a first step, we consider the polarization properties of the eigenmodes of the individual nanoparticle waveguides. Dispersion properties of such waveguides are discussed in details, e.g. in the Ref. [19]. Here, in Figs. 2(a,b) we show the schematic representation of the magnetic field polarization that can be understood within the framework of the dipole model. In this approximation all particles are modelled as point magnetic dipoles [19], with their instantaneous directions indicated by the arrows inside the circles at points "O". Such approximation can be justified for particles that possess only magnetic dipole resonant response, which is often the case, e.g. for silicon nanoparticles with typical linear size $\approx 100-300 \mathrm{~nm}$ in visible and near infrared frequency range [25]. Although, the resonant frequencies of high-index dielectric nanoparticles depend on their shapes and in some specific cases high-order multipoles should be taken into account [26], there is a wide range of parameters when the frequency of the fundamental magnetic dipole resonance is well separated from the higher-order ones, which in turn can be neglected. For instance, such approximation can be considered as relatively accurate even in the simplest case of spherical particles. In the dipole model two modes polarized in $x-y$ plane can be distinguished: $x$-polarized mode corresponds to the oscillation of magnetic dipoles along the waveguide axis and $y$-polarized mode correspond to the transverse oscillations of the dipoles. In what follows, we consider the waveguide modes close to the edge of the Brillouin zone, i.e. with Bloch wavenumber in $x$ direction close to $\beta_{x}=\pi$. In this case the fields in neighbor particles (or the neighboring dipoles) oscillate out-of-phase.

If we want to establish the polarization of the magnetic field at the points " $\mathrm{B}$ " or " $\mathrm{B}$ ", which are shifted along the $y$ axis from the center of the particle (point "O"), we need to add the contributions from all dipoles in the chain. In general, all dipoles except the nearest one numbered as $j=0$ induce both $x$ and $y$ components of the magnetic field at the points " $\mathrm{B}$ " and " $\mathrm{B}$ ". However, the contributions of the $y(x)$ component from the pair of dipoles with number $+j$ and $-j$ cancel each other in the case of $x$-polarized ( $y$-polarized) eigenmodes, respectively, because they oscillate in phase. Therefore, at these points magnetic field of the $x$-polarized $(y)$ mode oscillates in $x$ ( $y$ ) direction. To estimate the phase of the magnetic field we recall that the full field produced by a dipole is given by the dyadic Green's function [27]. Since it decreases with the distance, in the zeroth approximation we can assume

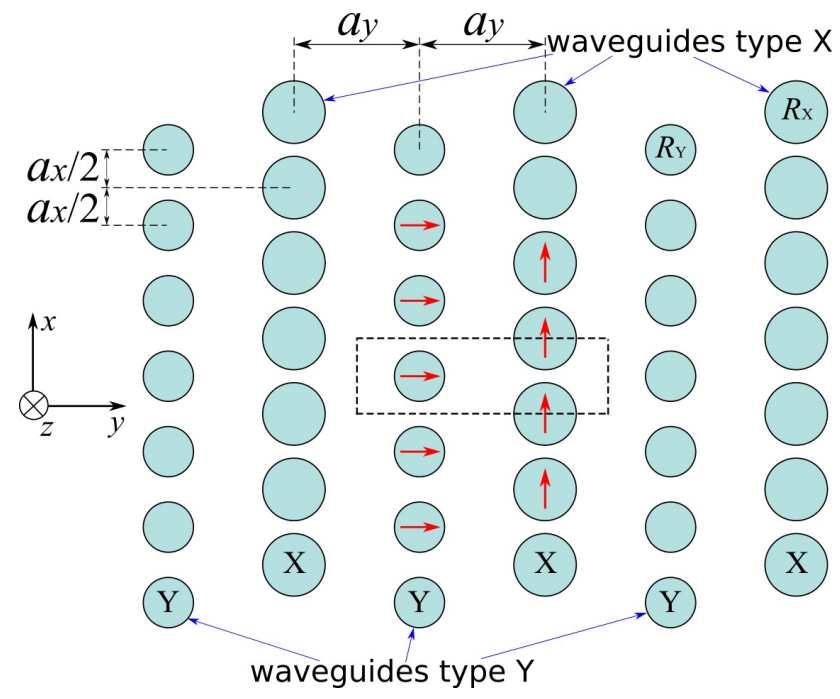

FIG. 1. A scheme of the array of periodic waveguides composed of dielectric nanoparticles with every other waveguide shifted in $x$ direction by $a_{x} / 2$. The distance between the waveguides is $a_{y}$. Waveguides denoted by "X" ("Y") are composed of dielectric cylinders with radius $R_{X}$ $\left(R_{Y}\right)$ and height $H$ (dimension along $z$ direction) placed with the period $a_{x}$. Polarization of the waveguide modes are determined by the magnetic field in the center of the cylinders. In the waveguides " $\mathrm{X}$ " ("Y") eigenmodes are polarized in $x(y)$ direction, which is indicated by the red arrows 
(a)
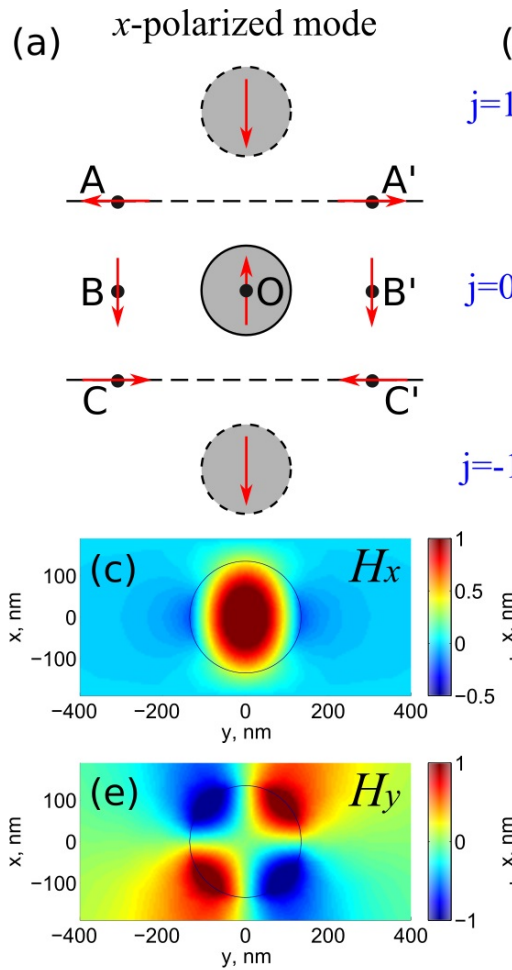

(b)

$y$-polarized mode
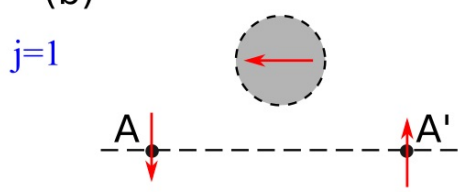

$\mathrm{j}=0$
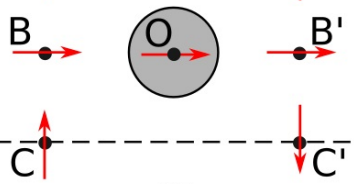

$j=-1$
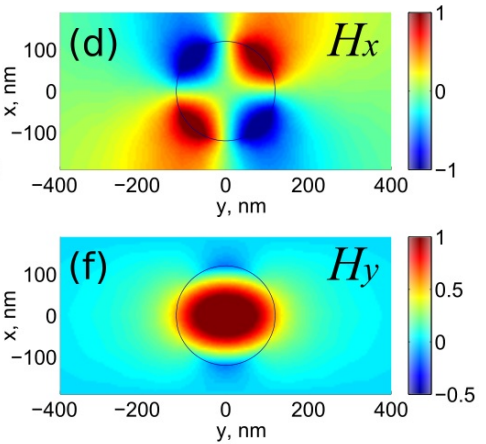

FIG. 2. (a,b) Schemes of the polarization of $x$ - and $y$-polarized modes, respectively. Red arrows indicate the direction of magnetic field. (c,d) Instantaneous $H_{x}$ and (e,f) $H_{y}$ components of the magnetic field distribution in chains of cylinders with (c,e) radius $R_{X}$ that exhibit $x$-polarized mode, and (d,f) radius $R_{Y}$ that exhibit $y$-polarized mode. Parameters of the waveguides are given in the text

that the largest contribution to the total field comes only from the nearest dipole. Further, we take into account only the near-field part of Green's function, which is $\propto 2 / r^{3}$ in the case of $x$ polarization and $\propto-1 / r^{3}$ in the case of $y$ polarization. This provides a qualitative explanation of why there is a zero phase difference between the magnetic field at points "B" " $\mathrm{B}$ "” and the dipole oscillations (indicated by the arrow at point "O") in Fig. 2(b), while for $x$-polarized mode in Fig. 2(a) the corresponding phase difference is equal to $\pi$.

The direction of the fields at points "A", " $\mathrm{A}$ " and "C", " $\mathrm{C}$ " can be analyzed in the same way. Now, the $x(y)$ component is cancelled for the $x(y)$ polarized mode. For instance, at the point "A" in Fig. 2(a) the $x$ component is cancelled because the dipole with numbers $j$ and $-j+1$ (e.g. 0 and 1) oscillate out-of-phase. Due to electric mirror symmetry with respect to $x-z$ plane we can immediately say that in Fig. 2(a) the field at the point " $\mathrm{A}$ " ("C'") should by oriented opposite to that at the point "A" ("C"). The same consideration applies to the $y$-polarized mode in Fig. 2(b) due to magnetic mirror symmetry.

To prove that this analysis is relevant to realistic systems, in Figs. 2(c-f) we show the numerically calculated magnetic field distribution of the eigenmodes of the chain of dielectric cylinders that correspond to Figs. 2(a,b). In Figs. 2(c,e) we show the field distribution in the chain of cylinders (one unit cell) with radius $R_{X}=135 \mathrm{~nm}$ and height $H=240 \mathrm{~nm}$ (dimension along $z$ direction) made from dielectric with permittivity $\varepsilon=13$ and placed with the period $a_{x}=380 \mathrm{~nm}$. The individual chain of such particles possesses $x$-polarized mode at the frequency $\omega_{0} \approx 2 \pi \cdot 290 \mathrm{rad} / \mathrm{s}$ with wavenumber $\beta_{x}=\pi$. Cylinders in Figs. 2(d,f) have slightly smaller radius $R_{Y}=120 \mathrm{~nm}$, so the chain of such cylinders supports $y$-polarized at approximately the same frequency $\omega_{0}$. We observe that the dipole model provides us with an adequate picture of the modes polarization for realistic parameters.

The conducted analysis and numerical calculations provide an intuitive picture of the coupling between the neighboring chains of nanoparticles. When there is no shift between the waveguides along $x$ direction, there is an interaction only between the waveguides of the same type, i.e. that support eigenmodes with the same polarization. When there is a non-zero shift, both $x$ and $y$ polarized waveguide modes can couple to each other, provided they have close eigenfrequencies. In the special case, when the waveguides are shifted by half of the period, the polarization in the points of the interest is rotated by $\pi / 2$ in the $x-y$ plane, as compared to the direction of 
oscillation of the field in the centers of particles. Therefore, there is an interaction only between the waveguide modes with different polarization.

\section{Couple mode theory for nanoparticle waveguides}

From the analysis of the polarization properties of the individual nanoparticle waveguide eigenmodes we can develop a simple model for the eigenmodes of the coupled array of the nanoparticle waveguides. If we assume that the waveguides are coupled only to their nearest neighbors, the system of equation appears as follows:

$$
\left\{\begin{array}{l}
\left(\omega_{0}-\omega\right) Y+\left(J_{1}+J_{2} e^{-i \beta_{y}}\right) X=0 \\
\left(\omega_{0}-\omega\right) X+\left(J_{1}+J_{2} e^{i \beta_{y}}\right) Y=0
\end{array}\right.
$$

where $\omega_{0}$ is the frequency of the eigenmodes of individual chains, $\omega$ is the frequency of the eigenmodes of the array of coupled waveguides, $\beta_{y}$ is the Bloch wavenumber along the $y$ axis, and coupling constants $J_{1}$ and $J_{2}$ are assumed to be real-valued, since there is no phase delay between the field at points " $O$ " and " $A$ ", " $C$ " in Figs. 2(a,b). In general, the system (1) is the celebrated SSH model [28,29], which is known to possess two bands separated by the bandgap when the coupling constants $J_{1}$ and $J_{2}$ are different. In this work, we restrict ourselves with the case when the distance between the waveguides is the same. As a consequence the coupling constants $J_{1}, J_{2}$ have the same magnitude and only differ in sign. Therefore, from the simple tight-binding model one cannot expect the formation of the gap in the spectrum of the array of equally spaced waveguides.

However, it is known that at the points close to the edge of the Brillouin zone, where the group velocity is small, coupling not only to the nearest neighbors might become substantial [30]. To estimate the effect caused by the long-range interaction we consider the model that takes into account also the coupling between next-to-nearest neighbors. Since the analysis of the eigenmodes polarization in Figs. 2(a,b) is valid for arbitrary $x$ coordinates of the points "A-C", we can expect that the waveguides $\mathrm{X}(\mathrm{Y})$ will couple not only to the nearest neighbor waveguides $\mathrm{Y}(\mathrm{X})$ but also to next-to-nearest neighbor waveguides $\mathrm{X}(\mathrm{Y})$ with a certain coupling constant $J_{X}\left(J_{Y}\right)$. Due to difference in the phase shift between the points "O" and "B" for different polarizations (see previous section) we expect coupling constants $J_{X}$ and $J_{Y}$ to have different signs. The values of $J_{X}$ and $J_{Y}$ in realistic systems may differ substantially from those expected from Figs. 2(c,f) mainly because we are interersted in fields at larger distances in $y$ direction. However, as we will see further, the main feature, which is the non-zero difference $\left|J_{X}-J_{Y}\right|$, remains unaffected.

The model that includes the interaction between the next-to-nearest neighbors appears as follows [31]:

$$
\left\{\begin{array}{l}
\left(\omega_{0}-\omega+2 J_{X} \cos \beta_{y}\right) X+\left(J_{1}+J_{2} e^{i \beta_{y}}\right) Y=0 \\
\left(\omega_{0}-\omega+2 J_{Y} \cos \beta_{y}\right) Y+\left(J_{1}+J_{2} e^{-i \beta_{y}}\right) X=0
\end{array}\right.
$$

Solution of the system (2) given the dispersion $\omega\left(\beta_{y}\right)$ :

$$
\frac{\omega=\omega_{0}+\left(J_{X}+J_{Y}\right) \cos \beta_{y} \pm}{\sqrt{\left(J_{X}-J_{Y}\right)^{2} \cos ^{2} \beta_{y}+J_{1}^{2}+J_{2}^{2}+2 J_{1} J_{2} \cos \beta_{y}}}
$$

Now, even when $\left|J_{1}\right|=\left|J_{2}\right|$ and there is no gap in the nearest neighbor approximation, the long-range interaction indeed induces the gap for non-equal constants $J_{X}$ and $J_{Y}$. For $\beta_{y}=0$ the spectral gap is given by $\Delta \omega_{c}=$ $2 \sqrt{\left(J_{X}-J_{Y}\right)^{2}+\left(J_{1}+J_{2}\right)^{2}}$, and for $\beta_{y}=\pi: \Delta \omega_{e}=2 \sqrt{\left(J_{X}-J_{Y}\right)^{2}+\left(J_{1}-J_{2}\right)^{2}}$. From that we can deduce that the system is gapped whenever the difference $\left|J_{X}-J_{Y}\right|$ is non-zero, which is exactly the case for the considered system. In a particular case when $J_{1}=-J_{2}=J$ we have $\Delta \omega_{c}=2\left|J_{X}-J_{Y}\right|$ and $\Delta \omega_{e}=2 \sqrt{\left(J_{X}-J_{Y}\right)^{2}+4 J^{2}}$.

As an example, in Fig. 3(a) we plot the dispersion for the two cases: $J_{X}=J_{Y}=0$ (solid black curves) and $J_{X}=-J_{Y}=0.4$ (dashed blue curves); other parameters are following: $\omega_{0}=0, J_{1}=-J_{2}=1$. We observe that the long-range interaction between the waveguides induces the gap in the eigenmodes spectrum.

It can be shown, that along with the existence of the gap in the infinite system for non-zero values of $\left|J_{X}-J_{Y}\right|$, in the finite size system one can also expect the formation of the edge states. The frequencies of these states $\omega_{e 1}$ and $\omega_{e 2}$ for the termination at "Y" or "X" site, respectively, can be estimated analytically as follows:

$$
\begin{aligned}
& \omega_{e 1} \approx \omega_{0}+2 J_{Y}\left[1-J_{Y}^{2} /\left(J^{2}+J_{Y}^{2}-J_{X} J_{Y}\right)\right], \\
& \omega_{e 2} \approx \omega_{0}+2 J_{X}\left[1-J_{X}^{2} /\left(J^{2}+J_{X}^{2}-J_{X} J_{Y}\right)\right] .
\end{aligned}
$$



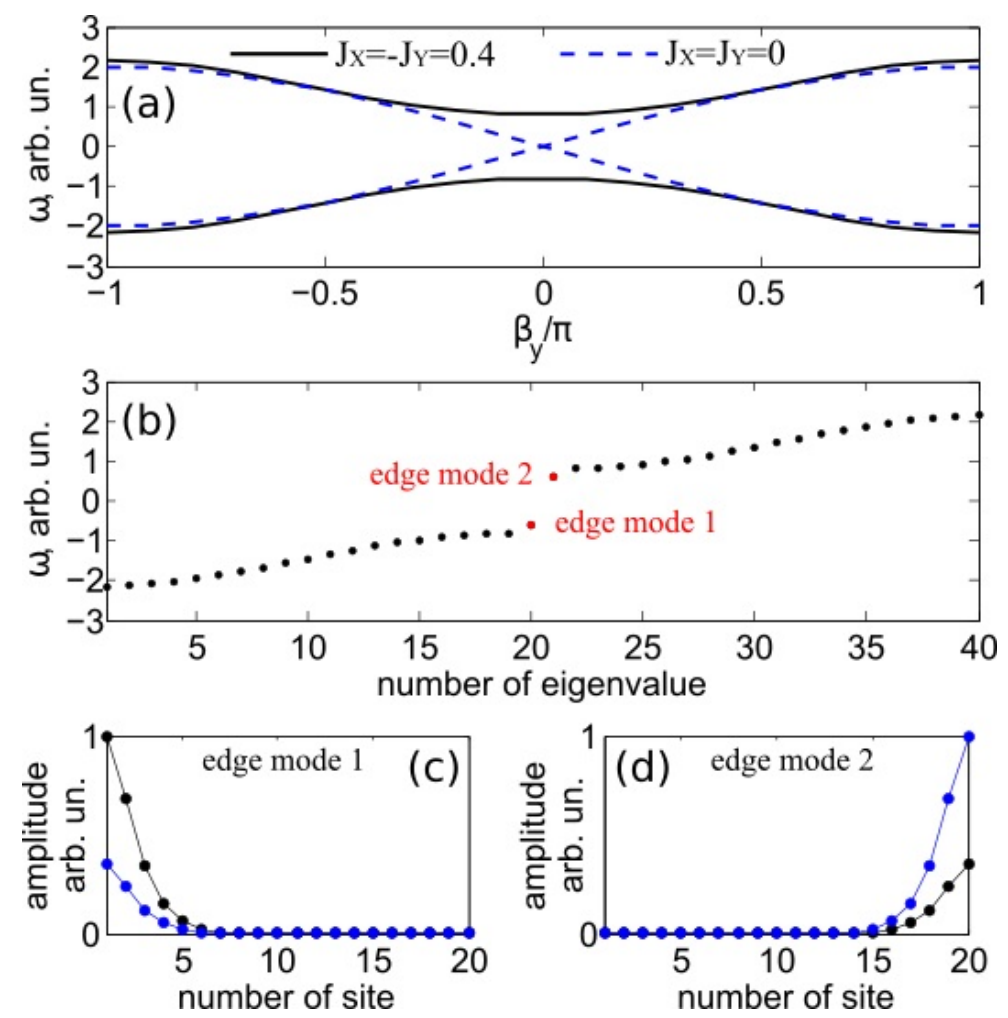

FIG. 3. (a) Spectrum of the eigenmodes calculated with equation (3) for the following parameters: $\omega_{0}=0, J_{1}=-J_{2}=1, J_{X}=J_{Y}=0$ (dashed blue curves), $J_{X}=-J_{Y}=0.4$ (solid black curves). (b) Eigenfrequencies of the finite size array consisting of 50 coupled chains for the parameters corresponding to solid black curves in (a). (c-d) Amplitudes of the edge modes marked in (b) as a function of the number of site; blue dots correspond to the amplitudes in sublattice $\mathrm{X}$ and black dots - in sublattice $\mathrm{Y}$

The amplitudes in two sublattices are related as $X / Y=J_{X} / J$ for the "X" edge termination and $X / Y=J_{Y} / J$ for "Y" edge termination. Further, since ratios $\left|J_{X, Y} / J\right|$ are assumed to be small we may simplify (4) as follows:

$$
\begin{aligned}
& \omega_{e 1}-\omega_{0}=2 J_{X}\left(1-J_{X}^{2} / J^{2}\right), \\
& \omega_{e 2}-\omega_{0}=2 J_{Y}\left(1-J_{Y}^{2} / J^{2}\right) .
\end{aligned}
$$

To verify our estimations, in Fig. 3(b) we plot the spectrum of the finite system consisted of the 20 unit cells (started with "A" on the right edge) for the same parameters as in Fig. 3(a) and $J_{X}=-J_{Y}=0.4$. We can observe that the finite array possesses two edge states symmetric with respect to $\omega_{0}$ (due to equality of the magnitudes of $J_{X}$ and $J_{Y}$ ) with the localization length that is determined by the proximity of the corresponding frequency to the central frequency $\omega_{0}$. The profiles of these states, shown in Fig. 3(c,d), also confirm the predicted ratios between the amplitude on the two sites within the unit cell.

\section{Results of numerical simulation of realistic systems}

Next, we study whether the long-range interaction is relevant in realistic systems. For that, we perform fullwave numerical simulations of the array of the cylinders with $z$-oriented axis in CST Microwave Studio software, which numerically solves Maxwell's equations for given geometrical and material parameters of the structure and boundary conditions. The parameters of the system were the same as in calculations in Fig. 2: for the sublattice $\mathrm{X}-R_{X}=135 \mathrm{~nm}$, for the sublattice $\mathrm{Y}-R_{Y}=120 \mathrm{~nm}$; height $H=240 \mathrm{~nm}, a_{x}=380 \mathrm{~nm}, a_{y}=450 \mathrm{~nm}$.

First, we calculate the spectrum of eigenmodes of the array infinite in both $x$ and $y$ directions. In this case periodic boundary conditions were employed in $x$ and $y$ directions, while in $z$ direction the electric walls were placed at the distance $\approx \lambda / 2$ from the array. Although, the system is not open in $z$ direction, this almost does not affect the modes that are localized near the array, due to exponential decay of the fields along $z$. Wavenumber in $x$ direction is fixed as $\beta_{x}=\pi$. In Fig. 4 we plot the eigenfrequency as a function of the wavenumber in $y$ direction $\beta_{y}$. These calculations qualitatively agree with those predicted in the next-to-nearest neighbors approximation: 


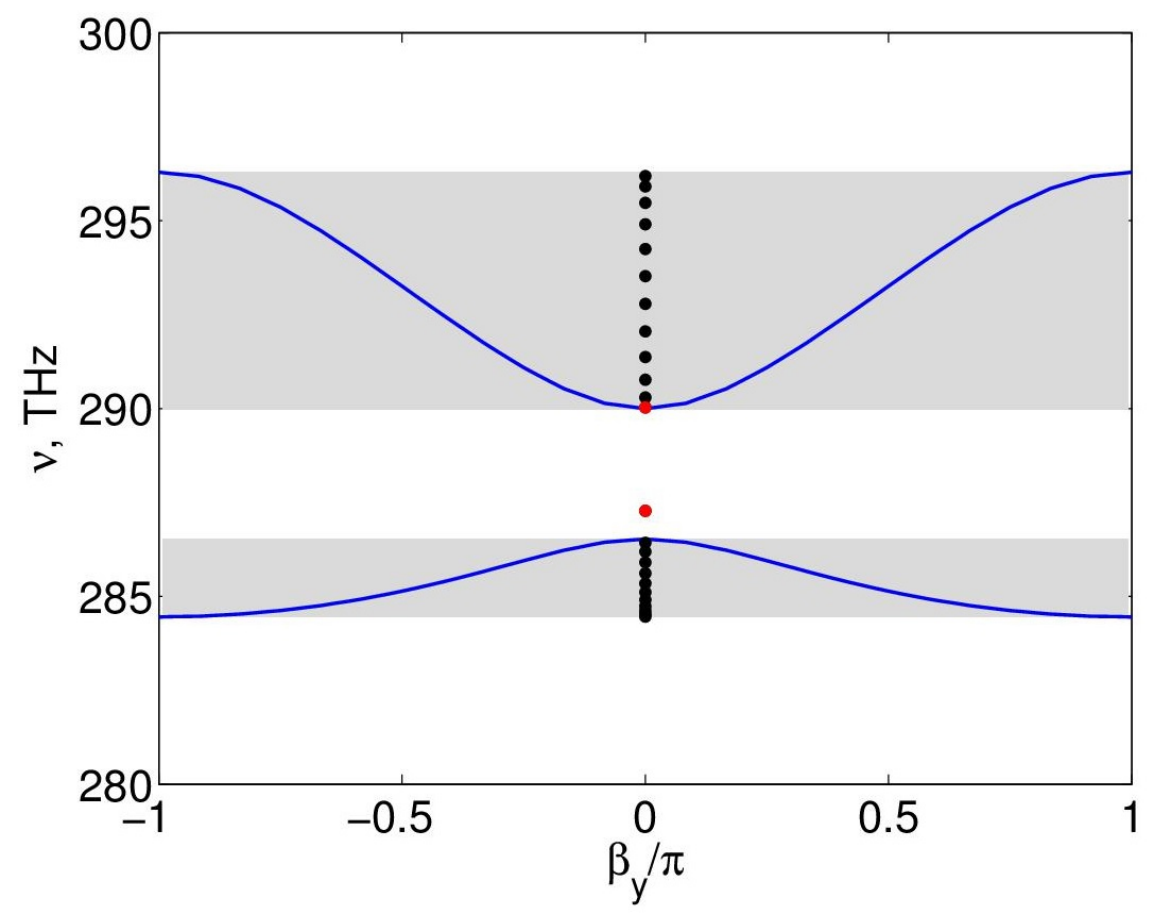

FIG. 4. Spectrum of the numerically calculated eigenmodes of an array of cylinders infinite in both $x$ and $y$ directions (solid blue curves) and infinite in $x$ direction and finite (12 periods) in $y$ direction (black and red dots). Wavenumber in $x$ direction is fixed as $\beta_{x}=\pi$. Red dots indicate the edge modes that fall in the gap region between the two bands (shaded gray areas)

there is a gap between two branches, and the difference between the frequencies of upper and lower branches is increasing when $\beta_{y}$ changes from 0 to $\pi$. We conclude that such system indeed can be described by taking into account at least next-to-nearest neighbor interaction, and therefore the finite size array should possess localized edge states.

To verify the existence of the edge states, we calculate the eigenmodes of the system in the strip geometry. More precisely, the system is infinite in $x$ direction with fixed $\beta_{x}=\pi$ and has $N$ periods in $y$ direction (see Fig. 1). Eigenfrequencies of the system with $N=12$ are shown in Fig. 5 with black and red dots. Black dots indicate the volume modes and are contained within the bands of the infinite structure, while red dots indicate the edge modes that fall within the gap. Note that the edge mode with higher frequency lies very close to the allowed band. This indicates that one of the interaction constants $J_{X}$ or $J_{Y}$ (in this case it is $J_{X}$ ) is in fact close to zero.

The magnetic field distribution of the edge modes within one unit cell in $x$ direction are shown in Figs. 5(a,b). The $x$ component of magnetic field at the centers of $\mathrm{X}$ cylinders and $y$ component of magnetic field at the centers of $\mathrm{Y}$ cylinders are shown in Fig. 5(c) with blue, magenta and black, red curves, respectively. We observe, that the localization strength and the ratio of the amplitudes in two sublattices is in well agreement with the predictions made in the previous section. Note that since the polarization of both sublattices is parallel to the plane of the array, the potential substrate inevitable in experiments would affect the properties of each chain in approximately the same way. Therefore, only slight adjustment of the geometrical parameters of the system would be necessary in the case when a substrate is present.

\section{Summary}

In summary, we have studied the properties of an array of coupled nanoparticle waveguides. We have shown that such structures may possess a spectral gap, the presence of which can be explained only by taking into account long range interaction between the waveguides. The appearance of the gap is accompanied by the formation of the defect-free edge modes in the finite size array. Our findings reveal the rich potential of the arrays of nanoparticle waveguides in realization of optical metasurfaces for guiding surface waves with complex functionalities and studying discrete systems beyond the tight-binding approximation. 
(a)
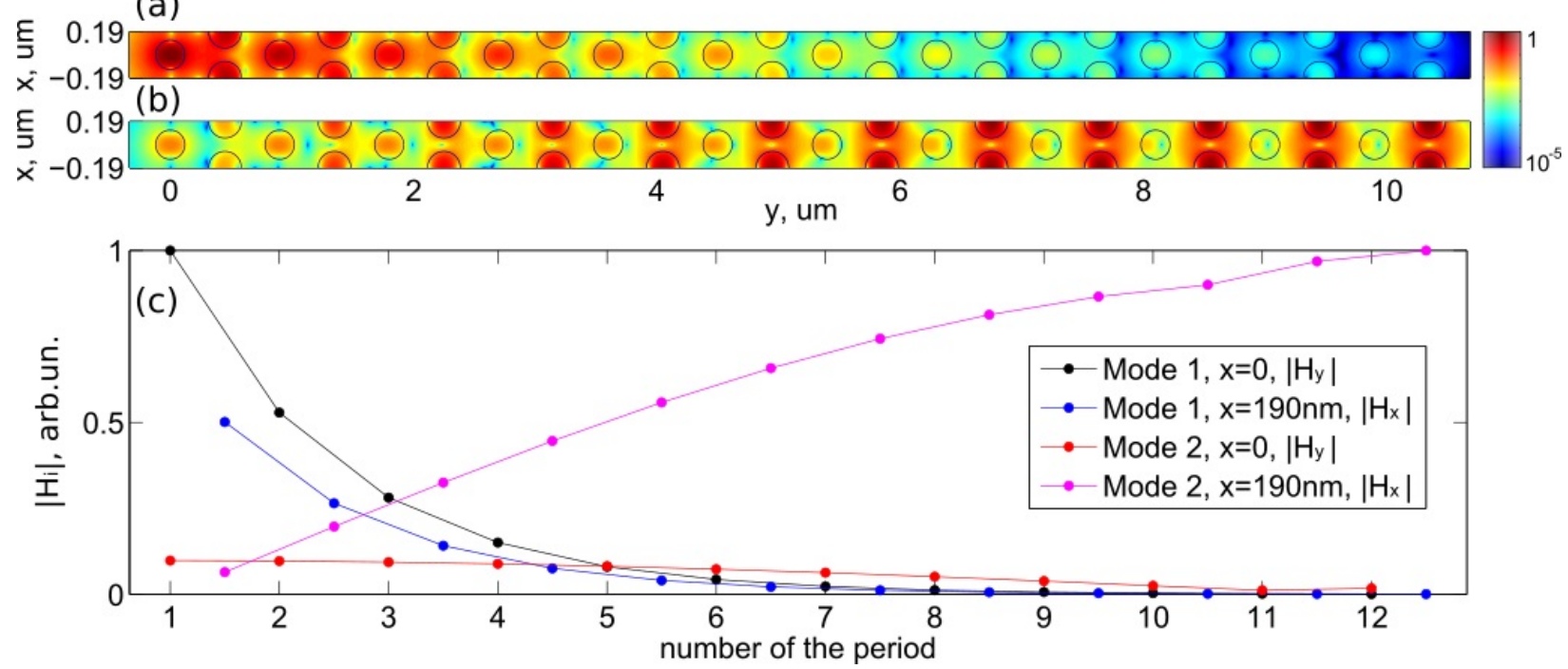

FIG. 5. (a,b) Magnetic field distribution $|H|$ of the two modes marked with red circles in Fig. 4 in the plane $z=0$ in logarithmic scale. The structure is periodic in $x$ direction; one unit cell is shown. (c) Amplitude of the $y$ component (black and red curves) and $x$ component (blue and magenta curves) of the magnetic field in the center of each cylinder; black and blue dots correspond to the edge mode in (a), red and magenta - in (b).

\section{Acknowledgements}

This work was supported by the Russian Foundation for Basic Research, according to the research project No. 16-37-60092 mol_a_dk, and the Grant from the President of the Russian Federation (MK-381.2017.2).

\section{References}

[1] Christodoulides D., Lederer F., Silberberg Y. Discretizing light behaviour in linear and nonlinear waveguide lattices. Nature, 2003, 424, P. 817-823.

[2] Malkova N., Hromada I., Wang X., Bryant G., Chen Z. Transition between Tamm-like and Shockleylike surface states in optically induced photonic superlattices. Phys. Rev. A., 2009, 80, P. 043806.

[3] Blanco-Redondo A., Andonegui I., Collins M.J., Harari G., Lumer Y., Rechtsman M.C., Eggleton B.J., Segev M. Topological Optical Waveguiding in Silicon and the Transition between Topological and Trivial Defect States. Phys. Rev. Lett. 2016, 116, P. 163901.

[4] Kraus Y.E., Lahini Y., Ringel Z., Verbin M., Zilberberg O. Topological States and Adiabatic Pumping in Quasicrystals. Phys. Rev. Lett., 2012, 109, P. 106402.

[5] Sukhorukov A.A., Kivshar Y.S., Eisenberg H.S., Silberberg Y. Spatial optical solitons in waveguide arrays. IEEE Journal of Quantum Electronics, 2003, 39, P. 31-50.

[6] Fleischer J.W., Bartal G., Cohen O., Schwartz T., Manela O., Freedman B., Segev M., Buljan H., Efremidis N.K. Spatial photonics in nonlinear waveguide arrays. Opt. Express, 2005, 13, P. 1780-1796.

[7] Peschel U., Pertsch T., Lederer F. Optical Bloch oscillations in waveguide arrays. Opt. Lett., 1998, 23, P. 1701-1703.

[8] Pertsch T., Dannberg P., Elflein W., Bräuer A., Lederer F. Optical Bloch Oscillations in Temperature Tuned Waveguide Arrays. Phys. Rev. Lett., 1999, 83, P. 4752-4755.

[9] Morandotti R., Peschel U., Aitchison J.S., Eisenberg H.S., Silberberg Y. Experimental Observation of Linear and Nonlinear Optical Bloch Oscillations. Phys. Rev. Lett., 1999, 83, P. 4756-4759.

[10] Longhi S. Optical Zener-Bloch oscillations in binary waveguide arrays. EPL (Europhysics Letters), 2006, 76, P. 416.

[11] Trompeter H., Krolikowski W., Neshev D.N., Desyatnikov A.S., Sukhorukov A.A., Kivshar Y.S., Pertsch T., Peschel U., Lederer F. Bloch Oscillations and Zener Tunneling in Two-Dimensional Photonic Lattices. Phys. Rev. Lett., 2006, 96, P. 053903.

[12] Lahini Y., Avidan A., Pozzi F., Sorel M., Morandotti R., Christodoulides D.N., Silberberg Y. Anderson Localization and Nonlinearity in One-Dimensional Disordered Photonic Lattices. Phys. Rev. Lett., 2008, 100, P. 013906.

[13] Martin L., Giuseppe G.D., Perez-Leija A., Keil R., Dreisow F., Heinrich M., Nolte S., Szameit A., Abouraddy A.F., Christodoulides D.N., Saleh B.E.A. Anderson localization in optical waveguide arrays with off-diagonal coupling disorder. Opt. Express, 2011, 19, P. $13636-13646$.

[14] Cherpakova Z., Bleckmann F., Vogler T., Linden S. Transverse Anderson localization of surface plasmon polaritons. Opt. Lett., 2017, 42, P. 2165-2168.

[15] Longhi S. Quantum-optical analogies using photonic structures. Laser and Photonics Reviews, 2009, 3(3), P. $243-261$.

[16] Garanovich I.L., Longhi S., Sukhorukov A.A., Kivshar Y.S. Light propagation and localization in modulated photonic lattices and waveguides. Phys. Rep., 2012, 518, P. 1-79. 
[17] Fan S., Winn J.N., Devenyi A., Chen J.C., Meade R.D., Joannopoulos J.D. Guided and defect modes in periodic dielectric waveguides. J. Opt. Soc. Am. B., 1995, 12, P. 1267-1272.

[18] Du J., Liu S., Lin Z., Zi J., Chui S.T. Dielectric-based extremely-low-loss subwavelength-light transport at the nanoscale: An alternative to surface-plasmon-mediated waveguiding. Phys. Rev. A, 2011, 83, P. 035803.

[19] Savelev R.S., Slobozhanyuk A.P., Miroshnichenko A.E., Kivshar Y.S., Belov P.A. Subwavelength waveguides composed of dielectric nanoparticles. Phys. Rev. B, 2014, 89, P. 035435.

[20] Bakker R.M., Yu Y.F., Paniagua-Domínguez R., Luk’yanchuk B., Kuznetsov A.I. Resonant Light Guiding Along a Chain of Silicon Nanoparticles. Nano Lett., 2017, 17, P. 3458-3464.

[21] Kuznetsov A.I., Miroshnichenko A.E., Brongersma M.L., Kivshar Y.S., Luk'yanchuk B. Optically resonant dielectric nanostructures. Science, 2016, 354.

[22] Savelev R.S., Filonov D.S., Kapitanova P.V., Krasnok A.E., Miroshnichenko A.E., Belov P.A., Kivshar Y.S. Bending of electromagnetic waves in all-dielectric particle array waveguides. Appl. Phys. Lett., 2014, 105, P. 181116.

[23] Kivshar Y.S. Nonlinear Tamm states and surface effects in periodic photonic structures. Laser Phys. Lett., 2008,5 , P. 703.

[24] Garanovich I.L., Sukhorukov A.A., Kivshar Y.S. Defect-Free Surface States in Modulated Photonic Lattices. Phys. Rev. Lett., 2008, 100, P. 203904.

[25] Evlyukhin A.B., Reinhardt C., Seidel A., Luk'yanchuk B.S., Chichkov B.N. Optical response features of Si-nanoparticle arrays. Phys. Rev. $B, 2010,82$, P. 045404.

[26] Evlyukhin A., Reinhardt C., Chichkov B. Multipole light scattering by nonspherical nanoparticles in the discrete dipole approximation. Phys. Rev. B, 2011, 23, P. 235429.

[27] Novotny L., Hecht B. Principles of Nano-optics. Cambridge University Press, New York, 2006.

[28] Su W.P., Schrieffer J.R., Heeger A.J. Solitons in Polyacetylene. Phys. Rev. Lett., 1979, 42, P. 1698-1701.

[29] Gorlach M., Slobozhanyuk A. Nonlinear topological states in the Su-Schrieffer-Heeger model. Nanosystems: Physics, Chemistry, Mathematics, 2017, 8, P. 695-700.

[30] Plotnik Y., Rechtsman M.C., Song D., Heinrich M., Zeuner J.M., Nolte S., Lumer Y., Malkova N., Xu J., Szameit A., Chen Z., Segev M. Observation of unconventional edge states in "photonic graphene". Nature Mater., 2013, 13, P. 57-62.

[31] Li L., Xu Z., Chen S. Topological phases of generalized Su-Schrieffer-Heeger models. Phys. Rev. B, 2014,89 , P. 085111. 